\title{
Increased external tibial torsion is an infratuberositary deformity and is not correlated with a lateralized position of the tibial tuberosity
}

\author{
Philipp W. Winkler ${ }^{1}$ Patricia M. Lutz ${ }^{1} \cdot$ Marco C. Rupp ${ }^{1} \cdot$ Florian B. Imhoff $^{1,2} \cdot$ Kaywan Izadpanah $^{3}$. \\ Andreas B. Imhoff ${ }^{1} \cdot$ Matthias J. Feucht ${ }^{1,3}$ (D
}

Received: 8 June 2020 / Accepted: 14 September 2020 / Published online: 25 September 2020

(c) The Author(s) 2020

\begin{abstract}
Purpose To perform a segmental analysis of tibial torsion in patients, with normal and increased external tibial torsion, suffering from chronic patellofemoral instability (PFI) and to investigate a possible correlation between tibial torsion and the position of the tibial tuberosity.

Methods Patients with chronic PFI who underwent torsional analysis of the lower limb using a standardized hip-knee-ankle MRI between 2016 and 2018 were included. For segmental analysis of tibial torsion, three axial levels were defined which divided the tibia into two segments: a distal, infratuberositary segment and a proximal, supratuberositary segment. Torsion was measured for the entire tibia (total tibial torsion, TTT), the proximal segment (proximal tibial torsion, PTT), and the distal segment (distal tibial torsion, DTT). Based on TTT, patients were assigned to one of two groups: Normal TTT $\left(<35^{\circ}\right)$ or increased external TTT $\left(>35^{\circ}\right)$. Position of the tibial tuberosity was assessed on conventional MRI scans by measuring the tibial tuberosity-trochlea groove (TT-TG) and the tibial tuberosity-posterior cruciate ligament (TT-PCL) distances.

Results Ninety-one patients ( $24 \pm 6$ years; $78 \%$ female) were included. Mean external TTT was $29.6^{\circ} \pm 9.1^{\circ}$ and 24 patients (26\%) had increased external TTT. Compared to patients with normal TTT, patients with increased external TTT demonstrated significantly higher values for DTT $\left(38^{\circ} \pm 8^{\circ}\right.$ vs. $\left.52^{\circ} \pm 9^{\circ} ; p<0.001\right)$, whereas no difference was found for PTT $\left(-13^{\circ} \pm 6^{\circ}\right.$ vs. $-12^{\circ} \pm 6^{\circ}$; n.s.). Furthermore, a significant correlation was found between TTT and DTT $(p<0.001)$, whereas no correlation was found between TTT and PTT (n.s). With regard to TT-TG and TT-PCL distances, no significant differences were observed between the two groups (TT-TG: $15 \pm 6$ vs. $14 \pm 4$ mm, n.s.; TT-PCL: $22 \pm 4$ vs. $21 \pm 5$ mm, n.s.) and no correlation was found with TTT, DTT, or PTT (n.s.).

Conclusion In patients with chronic PFI, increased external TTT of greater than $35^{\circ}$ is an infratuberositary deformity and does not correlate with a lateralized position of the tibial tuberosity.

Level of evidence Level III.
\end{abstract}

Keywords Tibial torsion $\cdot$ Segmental analysis $\cdot$ Patellofemoral instability $\cdot$ Tibial tubercle $\cdot$ Rotational alignment $\cdot$ Risk factor · Torsional osteotomy

The research was performed at the Department for Orthopedic Sports Medicine, Technical University Munich, Germany.

Matthias J. Feucht

matthias.feucht@gmx.net

1 Department for Orthopaedic Sports Medicine, Technical University Munich, Ismaninger Str. 22, 81675 Munich, Germany

2 Department of Orthopaedics, Balgrist University Hospital, University of Zurich, Zürich, Switzerland

3 Department of Orthopaedics and Trauma Surgery, Medical Center, Faculty of Medicine, Albert-Ludwigs-University of Freiburg, Freiburg, Germany

\section{Introduction}

Alignment, tracking, and stability of the patellofemoral joint depends on complex interactions between dynamic muscle action [23], passive soft tissue restraints [32], the surface geometry of the patella and trochlea, and limb alignment [2, 12, 16, 24, 43]. Therefore, patellofemoral dysfunction, such as patellofemoral instability, is commonly seen as a multifactorial problem [24, 43].

There is growing evidence that bony geometry and limb alignment play a major role in various patellofemoral disorders. Trochlear dysplasia and a lateralized tibial tuberosity 
are well accepted risk factors for chronic patellofemoral instability $[2,16,24,29,34,43]$. The role of valgus malalignment $[8,12,20,36,48]$ and torsional deformities $[4,7$, $11,13,19,37,42]$ are less understood. However, there is growing evidence that both, valgus and torsional malalignment promote patellar maltracking and instability $[9,15$, $28,30,35]$. Although associated with various patellofemoral disorders, such as anterior knee pain and patellofemoral instability [10, 11, 13, 19, 31, 37, 42], increased external tibial torsion is probably the least studied and hence least understood factor in patellofemoral dysfunction. Furthermore, little is known about the torsional geometry of the tibia $[14,18,21,22,27,38,39,45,47]$. From a biomechanical point of view, it is relevant whether the main torsional deformity is located proximally or distally to the tibial tuberosity. If the deformity is located proximally, increased external torsion would also result in a lateralized tuberosity and hence an increased $Q$ angle [5]. On the other hand, a distal deformity below the tuberosity may have only little to no impact on static patellofemoral alignment.

Despite the lack of knowledge about tibial torsional geometry, osteotomies of the tibia have been described to correct external tibial torsion and good clinical results have been reported [10, 11, 13, 19, 37, 42]. Nevertheless, it remains unclear whether the osteotomy should be performed below or above the tibial tuberosity. An osteotomy performed proximal to the tibial tuberosity will also alter the position of the tuberosity. It is therefore important to precisely define the association of tibial torsion and the position of the tibial tuberosity to determine the height of torsional osteotomies.

The purpose of the present study was to conduct a segmental analysis of tibial torsion in subjects with normal and increased external tibial torsion suffering from patellofemoral instability and to investigate a possible correlation between tibial torsion and the position of the tibial tuberosity. The hypotheses were that increased external tibial torsion is an infratuberositary deformity and is not associated with a lateralized position of the tibial tuberosity.

\section{Material and methods}

This retrospective cohort study was conducted to evaluate the torsional morphology of the tibia and its association with the position of the tibial tuberosity in patients suffering from chronic patellofemoral instability (PFI). This study was approved by the Ethics Committee of the Technical University of Munich (Nr.: 579/19 S).

Patients screened for eligibility had a history of recurrent patellar dislocations and a positive patellar apprehension test during clinical examination. Furthermore, torsional analysis of the lower limb using a standardized hip-knee-ankle
MRI (1.5 T, 8-mm slice thickness) at the authors' institution between 2016 and 2018 was required for inclusion. Torsional MRI was performed as part of a routine radiographic workup in all patients undergoing operative treatment for chronic PFI. Exclusion criteria for the present study were: skeletal immaturity, a history of lower extremity fracture or a history of surgical procedures affecting bony alignment, and presence of metal implants with artefacts on MR images or motion artifacts.

Clinical notes of all patients were reviewed to collect demographic data. All hip-knee-ankle MRI scans and conventional MRI scans were transferred from the institutions electronic picture archiving and communication system (PACS) to the OsiriX imaging software (https://www.osiri $\mathrm{X}$-viewer.com, Bernex, Switzerland), which was used to measure tibial torsion at different segments and lateralization of the tibial tuberosity as described in detail below. All measurements were obtained by the main observer (P.W.W.). For 20 randomly assigned patients, measurements were taken twice by the main observer (P.W.W.) at an interval of 6 weeks and once by a second observer (P.M.L.) to determine inter- and intrarater reliability.

\section{Segmental analysis of tibial torsion}

The described method is a further development of previously established and published methods to assess tibial torsion on axial MRI scans [12,39]. For the purpose of segmental analysis, three axial levels were defined which divided the tibia into two segments: a distal, infratuberositary segment and a proximal, supratuberositary segment (Fig. 1). Level 1 (L1) was defined as the axial slice just proximal to the apex of the fibular head [12, 39]. Level 2 (L2) was defined as the axial slice where the insertion of the patellar tendon at the tibial tuberosity could best be visualized. Level 3 (L3) was defined as the axial slice, where the talar articular surface and the medial and lateral malleolus could best be visualized $[12,39]$. The proximal (supratuberositary) segment was defined as the section between L1 and L2, and the distal (infratuberositary) segment was defined as the section between L2 and L3 (Fig. 1).

Total tibial torsion (TTT) was measured between L1 and L3 as previously described [12, 39]: Reference lines were drawn in L1 tangential to the posterior border of the tibial plateau and in L3 through the centers of the medial and lateral malleolus. TTT was defined as the angle between reference lines in L1 and L3 (Fig. 1). To measure proximal (supratuberositary) and distal (infratuberositary) tibial torsion, a third reference line was drawn in L2 tangential to the posterior tibial cortex. Proximal tibial torsion (PTT) was defined as the angle between the reference lines in L1 and L2, and distal tibial torsion (DTT) was defined as the angle between the reference lines in L2 and L3. For all torsional 


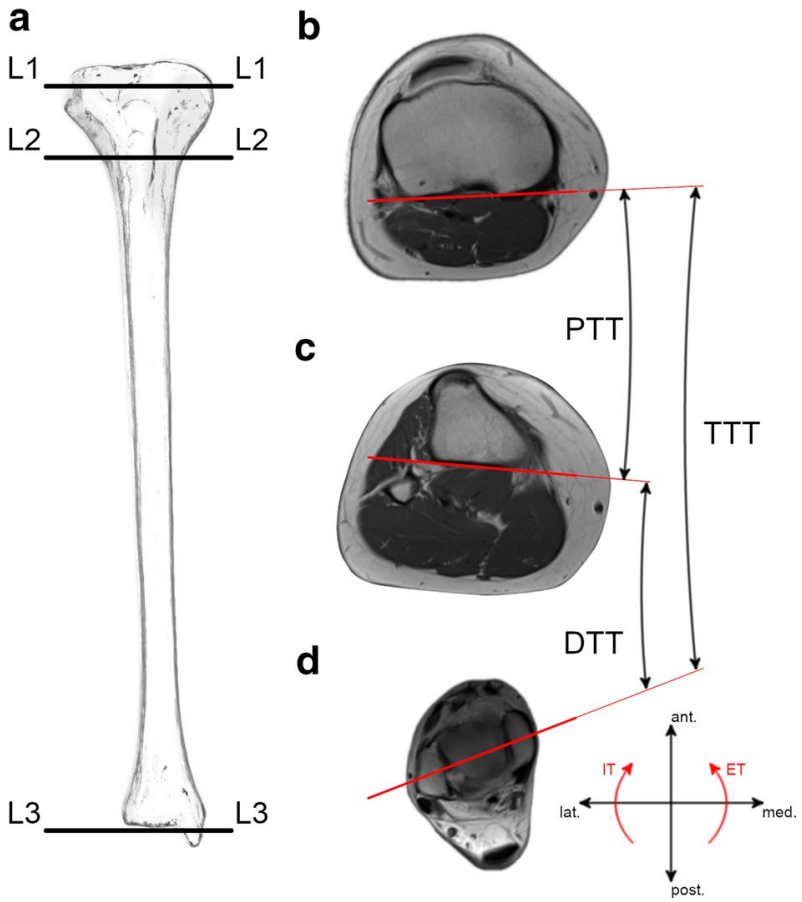

Fig. 1 Segmental analysis of tibial torsion. a Schematic illustration of a right tibia showing the levels of measurement (L1, L2, L3); b L1: Reference line tangential to the posterior border of the tibial plateau; c L2: Reference line tangential to the posterior tibial cortex; d L3: Reference line through the centers of the medial and lateral malleolus. Total tibial torsion (TTT): angle between reference lines in L1 and L3; proximal tibial torsion (PTT): angle between reference lines in L1 and L2; distal tibial torsion (DTT): angle between reference lines in L2 and L3; ant., anterior; ET, external torsion; IT, internal torsion; lat., lateral; med., medial; post., posterior

measurements, positive values indicate external tibial torsion and negative values indicate internal tibial torsion.

Based on the amount of TTT, patients were assigned to one of two groups: Normal tibial torsion $\left(<35^{\circ}\right)$ and increased external tibial torsion $\left(>35^{\circ}\right)$ [45]. To date, no consensus exists for normal and pathological tibial torsion, respectively. Based on a previously published study investigating tibial torsion on 504 intact tibiae in healthy volunteers, the cut-off value from normal to increased external TTT was set at $35^{\circ}$ [45].

\section{Position of the tibial tuberosity}

To analyze the position of the tibial tuberosity, the tibial tuberosity-trochlea groove (TT-TG) distance, and the tibial tuberosity-posterior cruciate ligament (TT-PCL) distance were measured on conventional MRI scans, as described by Schoettle et al. [40] and Seitlinger et al. [41], respectively. The TT-TG distance was measured on axial MRI images as the mediolateral distance between the midpoint of the insertion of the patellar tendon and the trochlear groove. The
TT-PCL distance was measured as the mediolateral distance between the midpoint of the insertion of the patellar tendon and the medial border of the tibial PCL attachment.

\section{Statistical analysis}

An a priori power analysis was conducted using the free available software G*Power (Erdfelder, Faul, Buchner, Lang, HHU Düsseldorf, Düsseldorf, Germany) [17]. According to previous studies, a mean external tibial torsion of $32^{\circ}$ and $40^{\circ}$ can be assumed for patients suffering from patellofemoral disorders [26] and torsional malalignment syndrome [3], respectively. An effect size of 0.8 was calculated based on a standard deviation of $10^{\circ}[3,26]$. An $\alpha$ of 0.05 and a ratio of 1:3 (increased TTT: normal TTT) resulted in a minimum total sample size of 54 subjects (13 with increased TTT, 41 with normal TTT) to achieve a statistical power of 0.8 .

Statistical analysis was performed using SPSS software version 25.0 (IBM-SPSS, New York, USA). Continuous variables were calculated as mean \pm standard deviation. Categorical variables were reported as count and percentages. Normal distribution of all data was evaluated with the Kolmogorov-Smirnov test. Group comparison (normal TTT vs. increased TTT) was performed with Chi-square test, Mann-Whitney $U$ test, and unpaired $t$ test, as appropriate. Correlation of continuous variables (TTT, PTT, DTT, TT-TG distance, and TT-PCL distance) was assessed with the Pearson correlation coefficient. The level of significance was set at $p<0.05$.

Intraclass correlation coefficients (ICCs) were calculated to determine the intra- and interobserver reproducibility. ICC values $>0.9$ were considered excellent, values between 0.8 and 0.9 were considered good and values $<0.8$ were considered poor.

\section{Results}

A total of 91 patients were included. Hip-Knee-Ankle MRI scans for segmental tibial torsion analysis were available for all patients and conventional MRI scans for TT-TG and TTPCL distance measurements were available for 62 patients (68\%).

Excellent intrarater reliability was observed for all measurements. The ICC values were 0.990, 0.981, 0.991, 0.998, and 0.998 for TT-TG, TT-PCL, TTT, DTT, and PTT, respectively. Interrater reliability was good to excellent with ICC values of $0.957,0.833,0.952,0.990$, and 0.983 for TT-TG, TT-PCL, TTT, DTT, and PTT, respectively.

Patient demographics and measurements of the total study group are shown in Table 1 . Values for TTT were positive in all patients, indicating tibial external torsion in all patients. With regard to the different tibial segments, the 
Table 1 Patient demographics and measurements of the total study group

\begin{tabular}{ll}
\hline Age (years) & $24.3 \pm 6.2(16-39)$ \\
Sex & \\
Male & $20(22 \%)$ \\
Female & $71(78 \%)$ \\
Laterality & \\
Right & $41(45 \%)$ \\
Left & $50(55 \%)$ \\
Tibial torsion & \\
Normal $\left(<35^{\circ}\right)$ & $67(74 \%)$ \\
Increased $\left(>35^{\circ}\right)$ & $24(26 \%)$ \\
Total tibial torsion $\left({ }^{\circ}\right)^{\mathrm{a}}$ & $29.6 \pm 9.1(6.0-63.2)$ \\
Proximal tibial torsion $\left({ }^{\circ}\right)^{\mathrm{a}}$ & $-12.4 \pm 5.6(-24.9-(-1.7))$ \\
Distal tibial torsion $\left({ }^{\circ}\right)^{\mathrm{a}}$ & $41.7 \pm 10.8(10.1-84.4)$ \\
TT-TG distance $(\mathrm{mm})^{\mathrm{b}}$ & $14.4 \pm 5.4(1.9-35.6)$ \\
TT-PCL distance $(\mathrm{mm})^{\mathrm{b}}$ & $21.3 \pm 4.5(7.9-29.5)$ \\
\hline
\end{tabular}

Continuous variables are shown as mean \pm standard deviation (range), categorical variables are shown as count and percentages

${ }^{a}$ Measurements were obtained in 91 patients (100\%); positive values indicate external torsion; negative values indicate internal torsion

${ }^{b}$ Measurements were obtained in 62 patients (68\%). TT-TG, tibial tuberosity-trochlea groove; TT-PCL, tibial tuberosity-posterior cruciate ligament

values for PTT were negative in all patients (internal torsion) and the values for DTT were positive in all patients (external torsion).

Increased external TTT $\left(>35^{\circ}\right)$ was observed in 24 patients, representing $26 \%$ of the total study group. No significant differences between patients with normal and increased TTT were found for sex (n.s.), laterality (n.s.), and age (n.s.). Group comparisons of the obtained measurements are shown in Table 2. With regard to tibial torsion, patients with an increased external TTT showed a significantly higher DTT, whereas no significant difference was observed for PTT (Fig. 2). No significant difference was observed for TT-TG and TT-PCL distances (Table 2) between the groups with normal and increased TTT.

The results of the correlation analysis are shown in Table 3. A statistically significant correlation was found between TTT and DTT, whereas no significant correlation was found between TTT and PTT (Fig. 3). TTT, PTT, and DTT did not correlate with TT-TG or TT-PCL distances (Table 3).

\section{Discussion}

The most important finding of the present study was that increased external TTT is an infratuberositary deformity. This finding was verified by two different statistical
Table 2 Group comparison between patients with normal and increased total tibial torsion

\begin{tabular}{|c|c|c|c|}
\hline & \multicolumn{2}{|l|}{ Tibial torsion } & \multirow[t]{2}{*}{$p$ value } \\
\hline & $\operatorname{Normal}\left(<35^{\circ}\right)$ & Increased $\left(>35^{\circ}\right)$ & \\
\hline $\begin{array}{l}\text { Total tibial torsion } \\
\left({ }^{\circ}\right)^{\mathrm{a}}\end{array}$ & $\begin{array}{l}25.6 \pm 6.4 \\
(6.0-35.0)\end{array}$ & $\begin{array}{l}40.8 \pm 5.7 \\
(35.8-63.2)\end{array}$ & $<0.001^{\mathrm{c}}$ \\
\hline $\begin{array}{l}\text { Proximal tibial tor- } \\
\text { sion }\left({ }^{\circ}\right)^{\mathrm{a}}\end{array}$ & $\begin{array}{l}-12.7 \pm 5.5 \\
(-24.9-(-2.0))\end{array}$ & $\begin{array}{l}-11.6 \pm 5.8 \\
(-21.2-(-1.7))\end{array}$ & n.s \\
\hline $\begin{array}{l}\text { Distal tibial torsion } \\
\left({ }^{\circ}\right)^{\mathrm{a}}\end{array}$ & $\begin{array}{l}37.9 \pm 8.4 \\
(10.1-58.7)\end{array}$ & $\begin{array}{l}52.4 \pm 9.4 \\
(42.3-84.4)\end{array}$ & $<0.001^{\mathrm{c}}$ \\
\hline $\begin{array}{l}\text { TT-TG distance } \\
(\mathrm{mm})^{\mathrm{b}}\end{array}$ & $\begin{array}{l}14.5 \pm 5.7 \\
(1.9-35.6)\end{array}$ & $\begin{array}{l}14.1 \pm 4.4 \\
(7.9-20.5)\end{array}$ & n.s \\
\hline $\begin{array}{l}\text { TT-PCL distance } \\
(\mathrm{mm})^{\mathrm{b}}\end{array}$ & $\begin{array}{l}21.5 \pm 4.4 \\
(7.9-29.5)\end{array}$ & $\begin{array}{l}20.6 \pm 4.8 \\
(11.8-26.0)\end{array}$ & n.s \\
\hline
\end{tabular}

Continuous variables are shown as mean \pm standard deviation (range)

${ }^{\mathrm{a}}$ Measurements were obtained in 91 patients (100\%); positive values indicate external torsion; negative values indicate internal torsion

${ }^{\mathrm{b}}$ Measurements were obtained in 62 patients (68\%)

${ }^{\mathrm{c}}$ Statistically significant difference between both groups $(p<0.05)$

$T T-T G$ tibial tuberosity-trochlea groove, TT-PCL tibial tuberosity-posterior cruciate ligament

approaches: First, no significant correlation was observed between TTT and PTT, whereas a significant correlation was observed between TTT and DTT. Second, compared to patients with normal TTT $\left(<35^{\circ}\right)$, patients with increased external TTT $\left(>35^{\circ}\right)$ showed a significantly higher DTT, whereas no significant difference was observed for PTT. These findings indicate that increased external TTT is primarily based on torsion of the distal (infratuberositary) segment. Another important finding was that tibial torsion is not correlated with the position of the tibial tuberosity. This finding was also verified by two different statistical approaches: First, no significant correlation was observed between TTT, PTT, or DTT and TT-TG or TT-PCL distances. Second, no
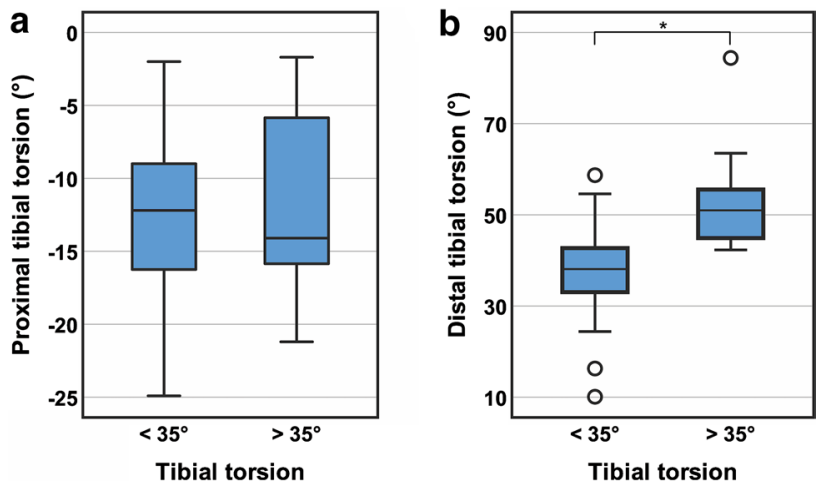

Fig. 2 Proximal and distal tibial torsion between patients with normal $\left(<35^{\circ}\right)$ and increased $\left(>35^{\circ}\right)$ total tibial torsion. a Group comparison of proximal tibial torsion: no significant difference, n.s.; b group comparison of distal tibial torsion: significant difference, $* p<0.001$ 
Table 3 Correlation analysis (Pearson)

\begin{tabular}{|c|c|c|c|c|c|}
\hline & Total tibial torsion & Proximal tibial torsion & Distal tibial torsion & TT-TG distance & TT-PCL distance \\
\hline Total tibial torsion & - & $\begin{array}{l}r=0.095 \\
\text { n.s }\end{array}$ & $\begin{array}{l}r=0.824 \\
p<0.001^{\mathrm{a}}\end{array}$ & $\begin{array}{l}r=-0.119 \\
\text { n.s }\end{array}$ & $\begin{array}{l}r=-0.063 \\
\text { n.s }\end{array}$ \\
\hline Proximal tibial torsion & $\begin{array}{l}r=0.095 \\
\text { n.s }\end{array}$ & - & $\begin{array}{l}r=-0.393 \\
p<0.001^{\mathrm{a}}\end{array}$ & $\begin{array}{l}r=0.010 \\
\text { n.s }\end{array}$ & $\begin{array}{l}r=-0.060 \\
\text { n.s }\end{array}$ \\
\hline Distal tibial torsion & $\begin{array}{l}r=0.824 \\
p<0.001^{\mathrm{a}}\end{array}$ & $\begin{array}{l}r=-0.393 \\
p<0.001^{\mathrm{a}}\end{array}$ & - & $\begin{array}{l}r=-0.142 \\
\text { n.s }\end{array}$ & $\begin{array}{l}r=-0.027 \\
\text { n.s }\end{array}$ \\
\hline TT-TG distance & $\begin{array}{l}r=-0.119 \\
\text { n.s }\end{array}$ & $\begin{array}{l}r=0.010 \\
\text { n.s }\end{array}$ & $\begin{array}{l}r=-0.142 \\
\text { n.s }\end{array}$ & - & $\begin{array}{l}r=0.511 \\
p<0.001^{\mathrm{a}}\end{array}$ \\
\hline TT-PCL distance & $\begin{array}{l}r=-0.063 \\
\text { n.s }\end{array}$ & $\begin{array}{l}r=-0.060 \\
\text { n.s }\end{array}$ & $\begin{array}{l}r=-0.027 \\
\text { n.s }\end{array}$ & $\begin{array}{l}r=0.511 \\
p<0.001^{\mathrm{a}}\end{array}$ & - \\
\hline
\end{tabular}

$r$ correlation coefficient, $p$ value, TT-TG tibial tuberosity-trochlea groove, TT-PCL tibial tuberosity-posterior cruciate ligament

${ }^{\text {a }}$ Statistically significant correlation $(p<0.05)$
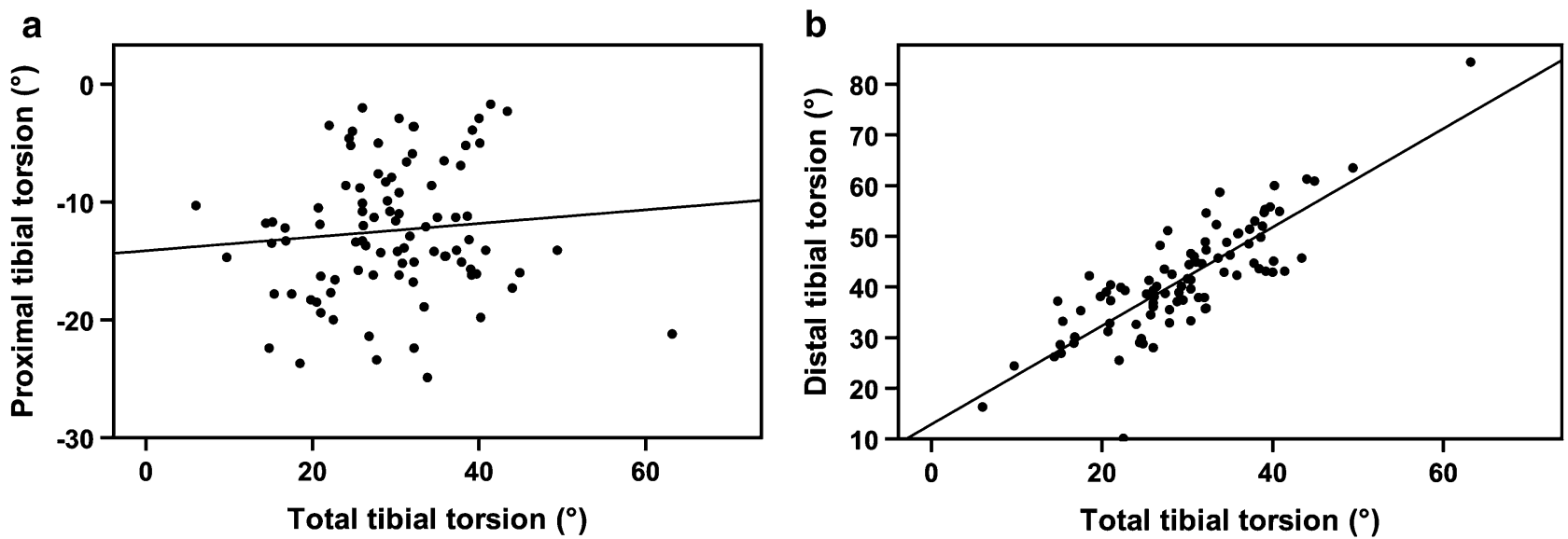

Fig. 3 Correlation between segmental and total tibial torsion. a No significant correlation between total tibial torsion and proximal tibial torsion $(r=0.095$, n.s. $)$; b significant correlation between total tibial torsion and distal tibial torsion $(r=0.824, p<0.001)$

significant differences were found for TT-TG and TT-PCL distances between patients with normal or increased external TTT.

Back in 1909, Le Damany [33] already investigated the torsional morphology of the tibia and described a mean external tibial torsion of $23.7^{\circ}$ based on anthropometric specimen examinations. Since then, several studies dealing with tibial torsion have been published [18, 22, 27, 38, 45, 47]. Considering the literature, a wide range of values for mean TTT becomes apparent, ranging from $20^{\circ}$ to $41.7^{\circ}$ [47]. A consensus exists regarding external direction of TTT [45, 47], as confirmed by the present study. The most extensive investigations for TTT analysis were performed by Strecker et al. [45] $(n=504)$ and Vanhove et al. [47] $(n=98)$, who observed a mean TTT of $34.9^{\circ} \pm 15.9^{\circ}$ and $25.5^{\circ}$ (left) $/ 27.7^{\circ}$ (right), respectively. This is consistent with the findings of the present study, in which tibial torsion analysis was performed on 91 patients and revealed a mean TTT of $29.6^{\circ} \pm 9.1^{\circ}$. However, detailed information on segmental tibial torsion and its impact on distal patellofemoral alignment is currently missing. Jakob et al. [27] were the only authors so far to investigate tibial torsion in different segments. Based on six cadaveric specimens, it has been shown that the greatest increase in tibial torsion is located in the proximal quarter of the tibial bone [27], which contradicts with the findings of the present study, in which the greatest increase in tibial torsion was found distal to the tibial tuberosity. In the present study, segmental tibial torsion was assessed in 91 patients with a highly reliable measurement technique based on torsional MRI. The applied measurement technique was a further development of previously established and published methods to assess TTT on axial MRI scans $[12,39]$. In contrast, the methodology of the study published by Jakob et al. exhibits some weaknesses owing to the small sample size and the lack of a precise definition of the applied levels of measurement [27].

Increased external tibial torsion, either in isolation or combined with increased femoral antetorsion, has been 
identified as one reason for various forms of patellofemoral dysfunction, including anterior knee pain and patellofemoral instability [5-7, 10, 11, 13, 15, 46]. Generally, tibial torsion is defined as the angulation between the most proximal and most distal part of the tibia around the longitudinal axis. Regarding the distal patellofemoral alignment, however, it is relevant whether the main torsional deformity is located proximally or distally to the tibial tuberosity. Based on the results of the present study, increased external tibial torsion can be regarded as an infratuberositary deformity. We therefore postulate that increased external tibial torsion has no relevant impact on the static distal patellofemoral alignment. This postulation is supported by a clinical study investigating the association between lower limb torsion and static patellar tracking in 59 patients with chronic PFI using torsional MR images. Although a statistically significant association between knee rotation and static patellar tracking has been demonstrated, no correlation between tibial torsion and static patellar tracking was observed [29]. Additionally, another clinical study showed no difference in tibial torsion between 30 patients with a history of patellar dislocation and an age- and sex-matched control group [12]. The distal patellofemoral alignment is influenced by the localization of the tibial tuberosity. The TT-TG and TT-PCL distances have become the most frequently used parameters to assess the position of the tibial tuberosity as indicators for distal patellofemoral realignment procedures (i.e., tibial tuberosity osteotomy) [25, 41]. It has been hypothesized that a lateralized tibial tuberosity may be caused by excessive external tibial torsion [13]. The present study, however, could not find a correlation between total or segmental tibial torsion and the TT-TG or TT-PCL distance. Furthermore, no differences in TT-TG and TT-PCL distances were observed between patients with normal or increased external tibial torsion. In accordance with other clinical studies $[3,12,29]$, the present findings provide further evidence that increased external tibial torsion is not associated with a lateralized position of the tibial tuberosity.

Although increased external tibial torsion has no relevant impact on static patellofemoral alignment, it may negatively affect the gait pattern. Increased external tibial torsion has been shown to result in compensatory gait deviations with increased hip internal rotation [1]. This adaption causes the knee to rotate internally leading to a lateral directed shear force on the patella. This dynamic impact of external tibial torsion may be the explanation for the good clinical results of torsional tibial osteotomies in patients with patellofemoral dysfunction $[4,5,11,13,19$, $37,42,44]$. Despite a growing number of clinical studies reporting outcomes after torsional tibial osteotomies, the level of the osteotomy (supratuberositary vs. infratuberositary) remains controversial. In the current literature, however, an osteotomy proximal to the tibial tuberosity seems to be preferred in patients with patellofemoral disorders $[11,37,42]$. It must be noted, however, that a supratuberositary torsional osteotomy will also result in medialization of the tibial tuberosity. Based on the results of the present study, which indicate that increased external tibial torsion is not associated with a lateralized tibial tuberosity, we suggest that the osteotomy height should be adjusted to the position of the tibial tuberosity: a supratuberositary osteotomy should be performed in patients with a lateralized tuberosity to prevent excessive medialization with subsequent overload of the medial patellofemoral compartment. In patients with normal position of the tibial tuberosity, an infratuberositary osteotomy should be considered. Thus, knowledge of the localization of a torsional deformity of the tibia in patients with increased external tibial torsion and chronic PFI may facilitate treatment decision-making (i.e., torsional tibial osteotomy, medialization of the tibial tuberosity).

Several limitations of this study have to be mentioned. First, there was no control group consisting of patients without patellofemoral disorders. Hip-Knee-Ankle MRI acquisition requires special indication and therefore was not economically affordable for a healthy control group. The contralateral tibiae were not investigated, because of significant physiological side-to-side differences described in the literature [38, 45, 47], which could have led to selection bias. Second, this study does not consider the dynamic behavior occurring in patients with torsional deformities. Only aspects concerning the statics of the patellofemoral joint were investigated. Third, conventional MRI was only available in 62 of the 91 patients in which the TT-TG and TT-PCL distances were measured.

\section{Conclusion}

In patients with chronic PFI, increased external TTT of greater than $35^{\circ}$ is an infratuberositary deformity and does not correlate with a lateralized position of the tibial tuberosity.

\section{Acknowledgements Not applicable.}

Author contributions All listed authors have contributed substantially to this work: P.W.W., M.J.F., K.I., A.B.I. for the study conception and design; P.W.W., P.M.L., M.C.R. for the data collection; P.W.W., M.J.F., F.B.I. for the data analysis; P.W.W., M.J.F., F.B.I, A.B.I., K.I. for the data interpretation; P.W.W., P.M.L., M.C.R. for the drafting of the manuscript, the figures, and the literature research; P.W.W., P.M.L., M.C.R., M.J.F., F.B.I, A.B.I., K.I. for critical revising the manuscript.

Funding Open Access funding enabled and organized by Projekt DEAL. 


\section{Compliance with ethical standards}

Conflict of interest Andreas B. Imhoff is a consultant for Arthrosurface and Medi Bayreuth and receives royalties from Arthrex and Arthrosurface.

Ethical approval This study was approved by the Ethics Committee of the Technical University of Munich (Nr.: 579/19 s).

Informed consent Not applicable.

Open Access This article is licensed under a Creative Commons Attribution 4.0 International License, which permits use, sharing, adaptation, distribution and reproduction in any medium or format, as long as you give appropriate credit to the original author(s) and the source, provide a link to the Creative Commons licence, and indicate if changes were made. The images or other third party material in this article are included in the article's Creative Commons licence, unless indicated otherwise in a credit line to the material. If material is not included in the article's Creative Commons licence and your intended use is not permitted by statutory regulation or exceeds the permitted use, you will need to obtain permission directly from the copyright holder. To view a copy of this licence, visit http://creativecommons.org/licenses/by/4.0/.

\section{References}

1. Alexander N, Wegener R, Lengnick H, Payne E, Klima H, Cip $\mathrm{J}$ et al (2020) Compensatory gait deviations in patients with increased outward tibial torsion pre and post tibial derotation osteotomy. Gait Posture 77:43-51

2. Balcarek P, Oberthur S, Hopfensitz S, Frosch S, Walde TA, Wachowski MM et al (2014) Which patellae are likely to redislocate? Knee Surg Sports Traumatol Arthrosc 22:2308-2314

3. Balcarek P, Radebold T, Schulz X, Vogel D (2019) Geometry of torsional malalignment syndrome: trochlear dysplasia but not torsion predicts lateral patellar instability. Orthop J Sports Med 7:2325967119829790

4. Bruce WD, Stevens PM (2004) Surgical correction of miserable malalignment syndrome. J Pediatr Orthop 24:392-396

5. Cameron JC, Saha S (1996) External tibial torsion: an underrecognized cause of recurrent patellar dislocation. Clin Orthop Relat Res 328:177-184

6. Cooke TD, Price N, Fisher B, Hedden D (1990) The inwardly pointing knee. An unrecognized problem of external rotational malalignment. Clin Orthop Relat Res 260:56-60

7. Delgado ED, Schoenecker PL, Rich MM, Capelli AM (1996) Treatment of severe torsional malalignment syndrome. J Pediatr Orthop 16:484-488

8. Dickschas J, Ferner F, Lutter C, Gelse K, Harrer J, Strecker W (2018) Patellofemoral dysbalance and genua valga: outcome after femoral varisation osteotomies. Arch Orthop Trauma Surg 138:19-25

9. Dickschas J, Harrer J, Bayer T, Schwitulla J, Strecker W (2016) Correlation of the tibial tuberosity-trochlear groove distance with the Q-angle. Knee Surg Sports Traumatol Arthrosc 24:915-920

10. Dickschas J, Harrer J, Pfefferkorn R, Strecker W (2012) Operative treatment of patellofemoral maltracking with torsional osteotomy. Arch Orthop Trauma Surg 132:289-298

11. Dickschas J, Tassika A, Lutter C, Harrer J, Strecker W (2017) Torsional osteotomies of the tibia in patellofemoral dysbalance. Arch Orthop Trauma Surg 137:179-185
12. Diederichs G, Kohlitz T, Kornaropoulos E, Heller MO, Vollnberg B, Scheffler S (2013) Magnetic resonance imaging analysis of rotational alignment in patients with patellar dislocations. Am J Sports Med 41:51-57

13. Drexler M, Dwyer T, Dolkart O, Goldstein Y, Steinberg EL, Chakravertty R et al (2014) Tibial rotational osteotomy and distal tuberosity transfer for patella subluxation secondary to excessive external tibial torsion: surgical technique and clinical outcome. Knee Surg Sports Traumatol Arthrosc 22:2682-2689

14. Eckhoff DG, Johnson KK (1994) Three-dimensional computed tomography reconstruction of tibial torsion. Clin Orthop Relat Res 302:42-46

15. Erkocak OF, Altan E, Altintas M, Turkmen F, Aydin BK, Bayar A (2016) Lower extremity rotational deformities and patellofemoral alignment parameters in patients with anterior knee pain. Knee Surg Sports Traumatol Arthrosc 24:3011-3020

16. Escala JS, Mellado JM, Olona M, Gine J, Sauri A, Neyret P (2006) Objective patellar instability: MR-based quantitative assessment of potentially associated anatomical features. Knee Surg Sports Traumatol Arthrosc 14:264-272

17. Faul F, Erdfelder E, Lang AG, Buchner A (2007) G*Power 3: a flexible statistical power analysis program for the social, behavioral, and biomedical sciences. Behav Res Methods 39:175-191

18. Forriol Campos F, Pascual Maiques JA (1990) The development of tibiofibular torsion. Surg Radiol Anat 12:109-112

19. Fouilleron N, Marchetti E, Autissier G, Gougeon F, Migaud H, Girard J (2010) Proximal tibial derotation osteotomy for torsional tibial deformities generating patello-femoral disorders. Orthop Traumatol Surg Res 96:785-792

20. Frings J, Krause M, Akoto R, Frosch KH (2019) Clinical results after combined distal femoral osteotomy in patients with patellar maltracking and recurrent dislocations. J Knee Surg 32:924-933

21. Gandhi S, Singla RK, Kullar JS, Agnihotri G, Mehta V, Suri RK et al (2014) Human tibial torsion-morphometric assessment and clinical relevance. Biomed J 37:10-13

22. Gaumetou E, Quijano S, Ilharreborde B, Presedo A, Thoreux P, Mazda K et al (2014) EOS analysis of lower extremity segmental torsion in children and young adults. Orthop Traumatol Surg Res 100:147-151

23. Gobbi RG, Hinckel BB, Teixeira PRL, Giglio PN, Lucarini BR, Pecora JR et al (2019) The vastus medialis insertion is more proximal and medial in patients with patellar instability: a magnetic resonance imaging case-control study. Orthop J Sports Med 7:2325967119880846

24. Hiemstra LA, Kerslake S, Lafave M (2017) Assessment of demographic and pathoanatomic risk factors in recurrent patellofemoral instability. Knee Surg Sports Traumatol Arthrosc 25:3849-3855

25. Hinckel BB, Gobbi RG, Kihara Filho EN, Demange MK, Pecora JR, Camanho GL (2015) Patellar tendon-trochlear groove angle measurement: a new method for patellofemoral rotational analyses. Orthop J Sports Med 3:2325967115601031

26. Imhoff FB, Funke V, Muench LN, Sauter A, Englmaier M, Woertler $\mathrm{K}$ et al (2020) The complexity of bony malalignment in patellofemoral disorders: femoral and tibial torsion, trochlear dysplasia, TT-TG distance, and frontal mechanical axis correlate with each other. Knee Surg Sports Traumatol Arthrosc 28:897-904

27. Jakob RP, Haertel M, Stussi E (1980) Tibial torsion calculated by computerised tomography and compared to other methods of measurement. J Bone Joint Surg Br 62-b:238-242

28. Kaiser P, Konschake M, Loth F, Plaikner M, Attal R, Liebensteiner $\mathrm{M}$ et al (2020) Derotational femoral osteotomy changes patella tilt, patella engagement and tibial tuberosity trochlear groove distance. Knee Surg Sports Traumatol Arthrosc 28:926-933

29. Kaiser P, Loth F, Attal R, Kummann M, Schuster P, Riechelmann $F$ et al (2020) Static patella tilt and axial engagement in knee 
extension are mainly influenced by knee torsion, the tibial tubercle-trochlear groove distance (TTTG), and trochlear dysplasia but not by femoral or tibial torsion. Knee Surg Sports Traumatol Arthrosc 28:952-959

30. Kaiser P, Schmoelz W, Schoettle P, Zwierzina M, Heinrichs C, Attal R (2017) Increased internal femoral torsion can be regarded as a risk factor for patellar instability - A biomechanical study. Clin Biomech (Bristol, Avon) 47:103-109

31. Krengel WF 3rd, Staheli LT (1992) Tibial rotational osteotomy for idiopathic torsion. A comparison of the proximal and distal osteotomy levels. Clin Orthop Relat Res 283:285-289

32. LaPrade MD, Kallenbach SL, Aman ZS, Moatshe G, Storaci HW, Turnbull TL et al (2018) Biomechanical evaluation of the medial stabilizers of the patella. Am J Sports Med 46:1575-1582

33. Le Damany P (1909) La torsion du tibia, normale et pathotogique, expérimentale. J Anat Physiol 45:598-615

34. Lewallen LW, McIntosh AL, Dahm DL (2013) Predictors of recurrent instability after acute patellofemoral dislocation in pediatric and adolescent patients. Am J Sports Med 41:575-581

35. Liska F, von Deimling C, Otto A, Willinger L, Kellner R, Imhoff $\mathrm{AB}$ et al (2019) Distal femoral torsional osteotomy increases the contact pressure of the medial patellofemoral joint in biomechanical analysis. Knee Surg Sports Traumatol Arthrosc 27:2328-2333

36. Nha KW, Ha Y, Oh S, Nikumbha VP, Kwon SK, Shin WJ et al (2018) Surgical treatment with closing-wedge distal femoral osteotomy for recurrent patellar dislocation with genu valgum. Am J Sports Med 46:1632-1640

37. Paulos L, Swanson SC, Stoddard GJ, Barber-Westin S (2009) Surgical correction of limb malalignment for instability of the patella: a comparison of 2 techniques. Am J Sports Med 37:1288-1300

38. Schenk P, Vlachopoulos L, Hingsammer A, Fucentese SF, Furnstahl P (2018) Is the contralateral tibia a reliable template for reconstruction: a three-dimensional anatomy cadaveric study. Knee Surg Sports Traumatol Arthrosc 26:2324-2331

39. Schneider B, Laubenberger J, Jemlich S, Groene K, Weber HM, Langer M (1997) Measurement of femoral antetorsion and tibial torsion by magnetic resonance imaging. Br J Radiol 70:575-579
40. Schoettle PB, Zanetti M, Seifert B, Pfirrmann CW, Fucentese SF, Romero J (2006) The tibial tuberosity-trochlear groove distance; a comparative study between CT and MRI scanning. Knee 13:26-31

41. Seitlinger G, Scheurecker G, Hogler R, Labey L, Innocenti B, Hofmann S (2012) Tibial tubercle-posterior cruciate ligament distance: a new measurement to define the position of the tibial tubercle in patients with patellar dislocation. Am J Sports Med 40:1119-1125

42. Server F, Miralles RC, Garcia E, Soler JM (1996) Medial rotational tibial osteotomy for patellar instability secondary to lateral tibial torsion. Int Orthop 20:153-158

43. Steensen RN, Bentley JC, Trinh TQ, Backes JR, Wiltfong RE (2015) The prevalence and combined prevalences of anatomic factors associated with recurrent patellar dislocation: a magnetic resonance imaging study. Am J Sports Med 43:921-927

44. Stevens PM, Gililland JM, Anderson LA, Mickelson JB, Nielson J, Klatt JW (2014) Success of torsional correction surgery after failed surgeries for patellofemoral pain and instability. Strategies Trauma Limb Reconstr 9:5-12

45. Strecker W, Keppler P, Gebhard F, Kinzl L (1997) Length and torsion of the lower limb. J Bone Joint Surg Br 79:1019-1023

46. Turner MS (1994) The association between tibial torsion and knee joint pathology. Clin Orthop Relat Res 302:47-51

47. Vanhove F, Noppe N, Fragomen AT, Hoekstra H, Vanderschueren G, Metsemakers WJ (2019) Standardization of torsional CT measurements of the lower limbs with threshold values for corrective osteotomy. Arch Orthop Trauma Surg 139:795-805

48. Wilson PL, Black SR, Ellis HB, Podeszwa DA (2018) Distal femoral valgus and recurrent traumatic patellar instability: is an isolated varus producing distal femoral osteotomy a treatment option? J Pediatr Orthop 38:e162-e167

Publisher's Note Springer Nature remains neutral with regard to jurisdictional claims in published maps and institutional affiliations. 